\title{
HYPERPLANE SECTIONS AND STABLE DERIVED CATEGORIES
}

\author{
KAZUSHI UEDA \\ (Communicated by Lev Borisov)
}

\begin{abstract}
We discuss the relation between the graded stable derived category of a hypersurface and that of its hyperplane section. The motivation comes from the compatibility between homological mirror symmetry for the CalabiYau manifold defined by an invertible polynomial and that for the singularity defined by the same polynomial.
\end{abstract}

\section{INTRODUCTION}

Let $\left(Y, \mathcal{O}_{Y}(1)\right)$ be a polarized smooth projective variety of dimension $d$ over a field $\boldsymbol{k}$, and $X=s^{-1}(0)$ be a smooth hypersurface defined by a section $s \in$ $H^{0}\left(\mathcal{O}_{Y}(a)\right)$ of degree $a$. For coherent sheaves $\mathcal{E}$ and $\mathcal{E}^{\prime}$ on $X$ restricted from those on $Y$, the push-forward functor $\iota_{*}: D^{b} \operatorname{coh} X \rightarrow D^{b} \operatorname{coh} Y$ along the inclusion $\iota: X \hookrightarrow Y$ satisfies

$$
\operatorname{Hom}_{Y}^{i}\left(\iota_{*} \mathcal{E}, \iota_{*} \mathcal{E}^{\prime}\right) \cong \operatorname{Hom}_{X}^{i}\left(\mathcal{E}, \mathcal{E}^{\prime}\right) \oplus \operatorname{Hom}_{X}^{d-i}\left(\mathcal{E}^{\prime},\left.\mathcal{E} \otimes \omega_{Y}\right|_{X}\right)^{\vee},
$$

where $\left.\omega_{Y}\right|_{X}=\iota^{*} \omega_{Y}$ is the restriction of the dualizing sheaf of $Y$ to $X$ and $\bullet \vee$ denotes the $\boldsymbol{k}$-dual vector space. In particular, if $Y$ has the trivial dualizing sheaf, then one has

$$
\operatorname{Hom}_{Y}^{i}\left(\iota_{*} \mathcal{E}, \iota_{*} \mathcal{E}^{\prime}\right) \cong \operatorname{Hom}_{X}^{i}\left(\mathcal{E}, \mathcal{E}^{\prime}\right) \oplus \operatorname{Hom}_{X}^{d-i}\left(\mathcal{E}^{\prime}, \mathcal{E}\right)^{\vee} .
$$

If $\omega_{Y} \cong \mathcal{O}_{Y}(r)$ for some $r \in \mathbb{Z}$ and $H^{i}\left(\mathcal{O}_{Y}(n)\right)=0$ for any $n \in \mathbb{Z}$ unless $i=0, d$, then the graded ring $\bar{S}=\bigoplus_{n=0}^{\infty} H^{0}\left(\mathcal{O}_{Y}(n)\right)$ is Gorenstein with a-invariant $r$, in the sense that $S$ has finite injective dimension as a graded $S$-module and the graded canonical module $K_{S}$ is isomorphic to the free module $S(r)$ [Orl09, Lemma 2.12]. The latter condition is equivalent to the isomorphism

$$
\mathbb{R H o m}_{\bar{S}}(\boldsymbol{k}, \bar{S}) \cong \boldsymbol{k}(-r)[-d-1] .
$$

Here the round bracket and the square bracket indicate the shift in the internal and homological grading respectively.

The graded stable derived category of $\bar{S}$ is defined as the quotient category

$$
D_{\text {sing }}^{b}(\operatorname{gr} \bar{S}):=D^{b}(\operatorname{gr} \bar{S}) / D^{\text {perf }}(\operatorname{gr} \bar{S})
$$

of the bounded derived category of finitely-generated graded $\bar{S}$-modules by the full triangulated subcategory $D^{\text {perf }}(\operatorname{gr} \bar{S})$ consisting of perfect complexes (i.e., bounded complexes of finitely-generated projective modules) [Buc87, Hap91, Kra05, Or104.

Received by the editors July 18, 2012 and, in revised form, October 2, 2012.

2010 Mathematics Subject Classification. Primary 13C14, 13D09; Secondary 14J33. 
Orlov Orl09, Theorem 2.13] has shown the existence of

- a full and faithful functor $\Psi_{\bar{S}}: D_{\text {sing }}^{b}(\operatorname{gr} \bar{S}) \hookrightarrow D^{b} \operatorname{coh} Y$ if $r<0$,

- an equivalence $\Psi_{\bar{S}}: D_{\text {sing }}^{b}(\operatorname{gr} \bar{S}) \stackrel{\sim}{\rightarrow} D^{b} \operatorname{coh} Y$ if $r=0$, and

- a full and faithful functor $\Psi_{\bar{S}}: D^{b} \operatorname{coh} Y \hookrightarrow D_{\text {sing }}^{b}(\operatorname{gr} \bar{S})$ if $r>0$.

The graded ring $\bar{R}=\bigoplus_{n=0}^{\infty} H^{0}\left(\mathcal{O}_{X}(n)\right)$ is the quotient ring of $\bar{S}$ by the principal ideal generated by $s$. Let $\Phi_{\mathrm{gr}}: \operatorname{gr} \bar{R} \rightarrow \operatorname{gr} \bar{S}$ be the functor sending a graded $\bar{R}$ module to the same module considered as a graded $\bar{S}$-module. Since $\bar{R}$ is perfect as an $\bar{S}$-module, the functor $\Phi_{\text {gr }}$ induces the push-forward functor

$$
\Phi_{\text {sing }}: D_{\text {sing }}^{b}(\operatorname{gr} \bar{R}) \rightarrow D_{\text {sing }}^{b}(\operatorname{gr} \bar{S})
$$

in the stable derived categories. When the $a$-invariant of $\bar{R}$ is 1 and $Y$ is Calabi-Yau, then KMU12, Theorem 1.1] states that the functor

$$
\Psi_{\bar{S}} \circ \Phi_{\text {sing }} \circ \Psi_{\bar{R}}: D^{b} \operatorname{coh} X \rightarrow D^{b} \operatorname{coh} Y
$$

is isomorphic to the push-forward functor

$$
\iota_{*}: D^{b} \operatorname{coh} X \rightarrow D^{b} \operatorname{coh} Y .
$$

In particular, if two objects $\mathcal{E}, \mathcal{E}^{\prime}$ in $D_{\text {sing }}^{b}(\operatorname{gr} \bar{R})$ are in the image of $\Psi_{\bar{R}} \circ \iota^{*}$ : $D^{b} \operatorname{coh} Y \rightarrow D_{\text {sing }}^{b}(\operatorname{gr} \bar{R})$, then (1.1) implies

$$
\operatorname{Hom}^{i}\left(\Phi_{\text {sing }}(\mathcal{E}), \Phi_{\text {sing }}\left(\mathcal{E}^{\prime}\right)\right) \cong \operatorname{Hom}^{i}\left(\mathcal{E}, \mathcal{E}^{\prime}\right) \oplus \operatorname{Hom}^{d-i}\left(\mathcal{E}^{\prime}, \mathcal{E}\right)^{\vee}
$$

On the other hand, the semiorthogonal complement of the image $\Psi_{\bar{R}}$ is generated by the structure sheaf $\bar{R} / \mathfrak{m}_{\bar{R}}$ of the origin, which goes to $\mathcal{O}_{Y}[d]$ by the composition $\Psi_{\bar{S}} \circ \Phi_{\text {sing. }}$. Since $\bar{R} / \mathfrak{m}_{\bar{R}}$ is exceptional and $\mathcal{O}_{Y}[d]$ is spherical, (1.2) holds also in this case.

In this paper, we prove the following graded stable derived category analog of (1.1): Let $R$ be a graded regular ring with $a$-invariant $r$ and Krull dimension $d$, and $S=R \otimes_{\boldsymbol{k}} \boldsymbol{k}[w]$ be the tensor product of $R$ with the polynomial ring in one variable $w$ of degree $a$. We identify the ring $R$ with its image by the natural injection $R \hookrightarrow S$. Further, let $f \in R_{h}$ be a homogeneous element of $R$ of degree $h$, and $F=f+w g \in S_{h}$ be a homogeneous element of $S$ of the same degree as $f$. We will always assume that $g \in w S$. The corresponding quotient rings will be denoted by $\bar{S}=S /(F)$ and $\bar{R}=R /(f) \cong \bar{S} /(w)$.

Theorem 1.1. For any objects $\mathcal{E}$ and $\mathcal{E}^{\prime}$ of $D_{\text {sing }}^{b}(\operatorname{gr} \bar{R})$, one has

$$
\operatorname{Hom}^{i}\left(\Phi_{\text {sing }}(\mathcal{E}), \Phi_{\text {sing }}\left(\mathcal{E}^{\prime}\right)\right) \cong \operatorname{Hom}^{i}\left(\mathcal{E}, \mathcal{E}^{\prime}\right) \oplus \operatorname{Hom}^{d-i}\left(\mathcal{E}^{\prime}, \mathcal{E}(r+h-a)\right)^{\vee} .
$$

In particular, when the $a$-invariant $r+h$ of $\bar{R}$ and the degree $a$ of the variable $w$ coincides (i.e., when $\bar{S}$ has the trivial canonical module), then one has

$$
\operatorname{Hom}^{i}\left(\Phi_{\text {sing }}(\mathcal{E}), \Phi_{\text {sing }}\left(\mathcal{E}^{\prime}\right)\right) \cong \operatorname{Hom}^{i}\left(\mathcal{E}, \mathcal{E}^{\prime}\right) \oplus \operatorname{Hom}^{d-i}\left(\mathcal{E}^{\prime}, \mathcal{E}\right)^{\vee}
$$

The motivation for Theorem 1.1 comes from the compatibility of homological mirror symmetry for the singularity defined by an invertible polynomial and that for the Calabi-Yau manifold defined by the same polynomial. An integer $(d+1) \times(d+1)$-matrix $\left(a_{i j}\right)_{i, j=1}^{d+1}$ with non-zero determinant defines a polynomial $f \in \boldsymbol{k}\left[x_{1}, \ldots, x_{d+1}\right]$ by

$$
f=\sum_{i=1}^{d+1} x_{1}^{a_{i 1}} \cdots x_{d+1}^{a_{i, d+1}},
$$


which is called invertible if it has an isolated critical point at the origin. Invertible polynomials play essential role in transposition mirror symmetry of Berglund and Hübsch BH93, which has attracted much attention recently (see e.g. Bor, CR11, $\mathrm{Kra}$ and the references therein). The quotient ring $\bar{R}=\boldsymbol{k}\left[x_{1}, \ldots, x_{d+1}\right] /(f)$ is naturally graded by the abelian group $L$ generated by $d+2$ elements $\vec{x}_{i}$ and $\vec{c}$ with relations

$$
a_{i 1} \vec{x}_{1}+\cdots+a_{i, d+1} \vec{x}_{d+1}=\vec{c}, \quad i=1, \ldots, d+1 .
$$

Homological mirror symmetry [Kon95] for invertible polynomials Tak10] is a conjectural equivalence

$$
D_{\text {sing }}^{b}(\operatorname{gr} \bar{R}) \cong D^{b} \mathfrak{F u k} \check{f}
$$

of triangulated categories. Here $\mathfrak{F} \mathfrak{u} \mathfrak{f}$ is the Fukaya-Seidel category Sei08] of the exact symplectic Lefschetz fibration obtained by Morsifying the Berglund-Hübsch transpose $\check{f}=\sum_{i=1}^{d+1} x_{1}^{a_{1 i}} \cdots x_{d+1}^{a_{d+1, i}}$ of $f$. The equivalence (1.4) is proved when $\check{f}$ is the Sebastiani-Thom sum of polynomials of types A or D [FU11, FU].

Assume that one can add one more term to $f$ and obtain another invertible polynomial,

$$
F=f+x_{1}^{a_{d+2,1}} \cdots x_{d+2}^{a_{d+2, d+2}} \in \boldsymbol{k}\left[x_{1}, \ldots, x_{d+2}\right],
$$

with a suitable $L$-grading on $x_{d+2}$ such that $F$ is homogeneous of degree $\vec{c}$ and the quotient ring $\bar{S}=\boldsymbol{k}\left[x_{1}, \ldots, x_{d+2}\right] /(F)$ is Gorenstein with the trivial $a$-invariant $K_{\bar{S}} \cong \bar{S}$.

The zero locus $F^{-1}(0)=\operatorname{Spec} \bar{S}$ has an action of $K=\operatorname{Hom}\left(L, \mathbb{G}_{m}\right)$ coming from the $L$-grading on $\bar{S}$, and the quotient stack $Y=\left[\left(F^{-1}(0) \backslash \mathbf{0}\right) / K\right]$ of the complement of the origin $\mathbf{0} \in F^{-1}(0)$ by this action is a Calabi-Yau orbifold. The Berglund-Hübsch transpose of $Y$ is another Calabi-Yau hypersurface $\check{Y}$ in a weighted projective space defined by the Berglund-Hübsch transpose $\breve{F}$ of $F$. It is conjectured BH93 that $(Y, \check{Y})$ is a mirror pair, so that there is an equivalence

$$
D^{b} \operatorname{coh} Y \cong D^{\pi} \mathfrak{F} \mathfrak{k} \grave{Y}
$$

of triangulated categories between the derived category of coherent sheaves on $Y$ and the split-closed derived Fukaya category of $\check{Y}$ Kon95. Sei11.

The weighted projective hypersurface $\check{Y}$ is a compactification of the Milnor fiber $\check{Y}^{\circ}=\check{Y} \backslash\left\{x_{d+2}=0\right\} \cong \check{f}^{-1}(-1)$ of $\check{f}$. Let $\left(V_{i}\right)_{i=1}^{m}$ be a distinguished basis of vanishing cycles of $\check{f}$, considered as Lagrangian submanifolds of $\check{Y}^{\circ}$. Further, let $\mathcal{F}^{\rightarrow}$ be the directed subcategory of $\mathfrak{F} \mathfrak{u} \mathfrak{Y} \check{Y}^{\circ}$ consisting of $\left(V_{i}\right)_{i=1}^{m}$; it is an $A_{\infty^{-}}$ subcategory with

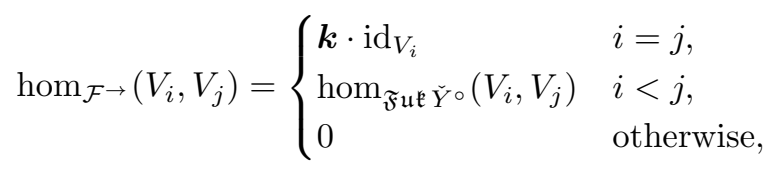

and $A_{\infty}$-operations on $\mathcal{F}^{\rightarrow}$ are inherited from those of $\mathfrak{F} \mathfrak{k} \mathfrak{K} \check{Y}^{\circ}$ in the obvious way. The Picard-Lefschetz theory [Sei08, Theorem 18.24] gives a derived equivalence

$$
D^{b} \mathcal{F}^{\rightarrow} \cong D^{b} \mathfrak{F} \mathfrak{u k} \check{f}
$$

of $\mathcal{F}^{\rightarrow}$ and the Fukaya-Seidel category of $\check{f}$. Although the Fukaya category $\mathfrak{F u k} \check{Y}$ is a deformation of $\mathfrak{F} \mathfrak{u k} \check{Y}^{\circ}$ Sei02] and the $A_{\infty}$-operations on $\mathfrak{F} \mathfrak{u k} \check{Y}$ is difficult to 
compute explicitly, Poincaré duality tells us that the space of morphisms in $\mathfrak{F} \mathfrak{u} \mathfrak{Y}$ is given by the Calabi-Yau completion

$$
\operatorname{Hom}_{\mathfrak{F} \mathfrak{u} \check{Y}}^{i}\left(V_{j}, V_{k}\right)=\operatorname{Hom}_{\mathcal{F} \rightarrow}^{i}\left(V_{j}, V_{k}\right) \oplus \operatorname{Hom}_{\mathcal{F} \rightarrow}^{d-i}\left(V_{k}, V_{j}\right)^{\vee}
$$

of the space of morphisms in $\mathcal{F}^{\rightarrow}$.

If we assume homological mirror symmetry (1.4) for singularities, then $D_{\text {sing }}^{b}(\mathrm{gr} \bar{R})$ has a full exceptional collection $\left(E_{i}\right)_{i=1}^{m}$ corresponding to $V_{i}$ and Theorem 1.1 shows that

- the full subcategory of $D_{\text {sing }}^{b}(\operatorname{gr} \bar{S}) \cong D^{b} \operatorname{coh} Y$ consisting of $\left(\Phi_{\text {sing }}\left(E_{i}\right)\right)_{i=1}^{m}$ is a Calabi-Yau completion of the full subcategory $\mathcal{E}^{\rightarrow}$ of $D_{\text {sing }}^{b}(\mathrm{gr} \bar{R})$ consisting of $\left(E_{i}\right)_{i=1}^{m}$ and

- $\mathcal{E}^{\rightarrow}$ is the directed subcategory of $D_{\text {sing }}^{b}(\operatorname{gr} \bar{S})$ consisting of $\left(\Phi_{\text {sing }}\left(E_{i}\right)\right)_{i=1}^{m}$.

Now the compatibility of homological mirror symmetry for Calabi-Yau manifolds and that for singularities is the existence of a commutative diagram

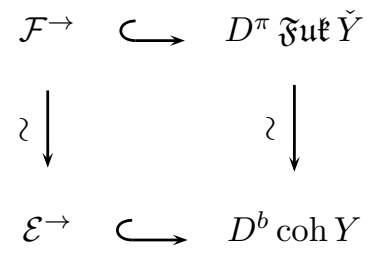

where horizontal arrows are embeddings of directed subcategories and vertical equivalences are homological mirror symmetry. Moreover, we expect that the images of the horizontal arrows split-generate the categories on the right so that one can divide the proof of homological mirror symmetry for Calabi-Yau manifolds into two steps: the first step is the proof of homological mirror symmetry for singularities, and the second step is the analysis of Calabi-Yau completion. This is analogous to the proof of homological mirror symmetry for Calabi-Yau hypersurfaces in projective spaces, which can be interpreted as first proving homological mirror symmetry for the ambient projective space, and then passing to the Calabi-Yau hypersurface by taking a non-trivial Calabi-Yau completion Sei11, She11, NU12].

This paper is organized as follows: In Section 2, we recall basic definitions on matrix factorizations. In Section 3. we give an explicit description of the pushforward in terms of matrix factorizations, which will be used in Section 4 to prove Theorem 1.1.

\section{Graded matrix FaCtorizations}

Let $R$ be a graded regular ring with $a$-invariant $r$ and Krull dimension $d$, and $f \in R_{h}$ be a homogeneous element of degree $h$. We assume that $R$ is graded by $\mathbb{Z}$ for simplicity of exposition, although our discussion easily generalizes to grading by any abelian group.

A graded matrix factorization

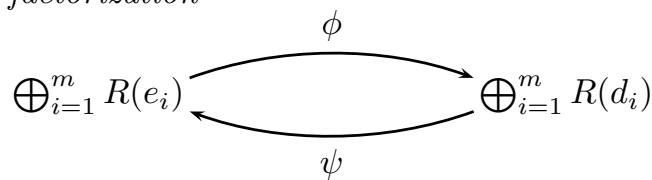

of $f$ over $R$ consists of

- a pair $M_{0}=\bigoplus_{i=1}^{m} R\left(d_{i}\right)$ and $M_{1}=\bigoplus_{i=1}^{m} R\left(e_{i}\right)$ of graded free $R$-module and 
- a pair $(\phi, \psi)$ of morphisms $\phi: M_{1} \rightarrow M_{0}$ and $\psi: M_{0}(-h) \rightarrow M_{1}$ of graded modules

satisfying $\phi \circ \psi=f \cdot \mathrm{id}_{M^{0}}$ and $\psi \circ \phi=f \cdot \mathrm{id}_{M^{1}}$. The integers $d_{i}$ and $e_{i}$ are shifts in the internal grading of $R$-modules, and the natural number $m=\operatorname{rank} M^{0}=\operatorname{rank} M^{1}$ is called the rank of the matrix factorization.

A morphism of graded matrix factorizations from $(\phi, \psi): M_{1} \rightleftarrows M_{0}$ to $\left(\phi^{\prime}, \psi^{\prime}\right)$ : $M_{1}^{\prime} \rightleftarrows M_{0}^{\prime}$ is a pair $(\alpha, \beta)$ of morphisms $\alpha: M_{0} \rightarrow M_{0}^{\prime}$ and $\beta: M_{1} \rightarrow M_{1}^{\prime}$ making the diagram

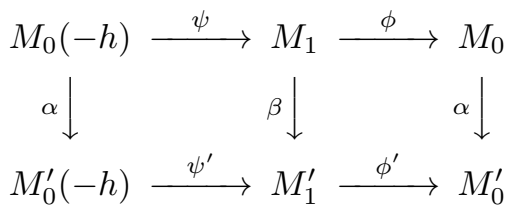

commute: $\alpha \circ \phi=\phi^{\prime} \circ \beta$ and $\beta \circ \psi=\psi^{\prime} \circ \alpha$. Here, the morphism $M_{0}(-h) \rightarrow M_{0}^{\prime}(-h)$ of graded $R$-modules corresponding to the morphism $\alpha: M_{0} \rightarrow M_{0}^{\prime}$ is denoted by the same symbol.

Two morphisms $(\alpha, \beta)$ and $\left(\alpha^{\prime}, \beta^{\prime}\right)$ are homotopic if there exist morphisms $\xi$ : $M_{0} \rightarrow M_{1}^{\prime}$ and $\eta: M_{1} \rightarrow M_{0}^{\prime}(-h)$ satisfying $\alpha-\alpha^{\prime}=\phi^{\prime} \circ \xi+\eta \circ \psi$ and $\beta-\beta^{\prime}=$ $\psi^{\prime} \circ \eta+\xi \circ \phi$.

The homotopy category of graded matrix factorizations of $f$ over $R$ is the category $\mathrm{Hmf}_{R}^{\mathrm{gr}} f$ whose objects are graded matrix factorizations of $f$ over $R$ and whose morphisms are morphisms of graded matrix factorizations up to homotopy.

The category $\operatorname{Hmf}_{R}^{\mathrm{gr}} f$ is equivalent to the stable category $\mathrm{CM}^{\mathrm{gr}} \bar{R}$ of graded Cohen-Macaulay modules over the quotient ring $\bar{R}=R /(f)$ Eis80, which in turn is equivalent to the graded stable derived category $D_{\text {sing }}^{b}(\operatorname{gr} \bar{R})$ of $\bar{R}$ [Buc87, Orl04].

\section{THE PUSH-FORWARD OF A MATRIX FACTORIZATION}

We keep the notation from Section 2, Let $S=R \otimes \boldsymbol{k}[w]$ be another graded ring with $\operatorname{deg} w=a$ and $F=f+w g$ be a homogeneous element of $S$ such that $g \in w S$. The corresponding quotient ring will be denoted by $\bar{S}=S /(F)$, and one has natural injections $R \hookrightarrow S$ and $\bar{R} \hookrightarrow \bar{S}$. The natural ring homomorphism $\bar{S} \rightarrow \bar{S} /(w) \cong \bar{R}$ induces the push-forward functor

$$
\Phi_{\text {sing }}: D_{\text {sing }}^{b}(\operatorname{gr} \bar{R}) \rightarrow D_{\text {sing }}^{b}(\operatorname{gr} \bar{S}),
$$

since $\bar{R}$ is perfect as an $\bar{S}$-module.

Proposition 3.1. The functor

$$
\Phi_{\mathrm{Hmf}}: \operatorname{Hmf}_{R}^{\mathrm{gr}} f \rightarrow \operatorname{Hmf}_{S}^{\mathrm{gr}} F
$$

corresponding to the push-forward functor

$$
\Phi_{\text {sing }}: D_{\text {sing }}^{b}(\operatorname{gr} \bar{R}) \rightarrow D_{\text {sing }}^{b}(\operatorname{gr} \bar{S})
$$

sends the graded matrix factorization

$$
\bigoplus_{i=1}^{m} R\left(d_{i}-h\right) \stackrel{\psi}{\longrightarrow} \bigoplus_{i=1}^{m} R\left(e_{i}\right) \stackrel{\phi}{\longrightarrow} \bigoplus_{i=1}^{m} R\left(d_{i}\right)
$$


of $f$ over $R$ to the graded matrix factorization

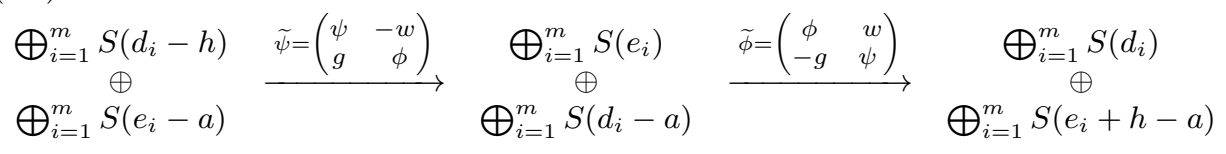

of $F$ over $S$, where $\phi, \psi \in R$ are considered as elements of $S$ by the injection $R \hookrightarrow S$.

Proof. Recall from [Eis80] that the matrix factorization (3.1) corresponds to the $\bar{R}$-module $\bar{M}=$ coker $\bar{\phi}$ through the 2 -periodic projective resolution

$$
\begin{aligned}
\cdots \stackrel{\bar{\psi}}{\longrightarrow} \bigoplus_{i=1}^{m} \bar{R}\left(e_{i}-h\right) \stackrel{\bar{\phi}}{\longrightarrow} \bigoplus_{i=1}^{m} \bar{R}\left(d_{i}-h\right) \stackrel{\bar{\psi}}{\longrightarrow} \bigoplus_{i=1}^{m} \bar{R}\left(e_{i}\right) & \\
\stackrel{\bar{\phi}}{\longrightarrow} \bigoplus_{i=1}^{m} \bar{R}\left(d_{i}\right) \longrightarrow \bar{M} \longrightarrow 0 & \longrightarrow \bar{M} \longrightarrow 0
\end{aligned}
$$

where $\bar{\psi}$ and $\bar{\phi}$ are morphisms of $\bar{R}$-modules induced by $\psi$ and $\phi$. By replacing each free $\bar{R}$-module in (3.3) with its $\bar{S}$-free resolution

$$
0 \rightarrow \bar{S}(\ell-a) \stackrel{w}{\rightarrow} \bar{S}(\ell) \rightarrow \bar{R}(\ell) \rightarrow 0,
$$

one obtains a projective resolution

$$
\begin{aligned}
& \cdots \stackrel{\tilde{\bar{\psi}}}{\longrightarrow} \begin{array}{lll}
\bigoplus_{i=1}^{m} \bar{S}\left(e_{i}-h\right) \\
\bigoplus_{i=1}^{m} \bar{S}\left(d_{i}-a-h\right),
\end{array} \stackrel{\tilde{\bar{\phi}}}{\longrightarrow} \begin{array}{l}
\bigoplus_{i=1}^{m} \bar{S}\left(d_{i}-h\right) \\
\bigoplus_{i=1}^{m} \bar{S}\left(e_{i}-a\right)
\end{array}
\end{aligned}
$$

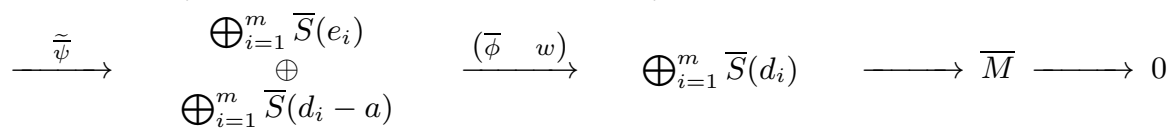

of $\bar{M}$ as an $\bar{S}$-module, where $\widetilde{\bar{\phi}}$ and $\widetilde{\bar{\psi}}$ are morphisms of $\bar{S}$-modules induced by $\widetilde{\phi}$ and $\widetilde{\psi}$. This complex is 2-periodic except for the first two terms and clearly corresponds to the matrix factorization (3.2), so that Proposition 3.1 is proved.

\section{Morphisms BetweEn PUSH-FORWARdS}

We keep the notation from Section 3. The following proposition gives Theorem 1.1 written in terms of matrix factorizations.

Proposition 4.1. Let $\mathcal{E}=(\phi, \psi)$ and $\mathcal{E}^{\prime}=\left(\phi^{\prime}, \psi^{\prime}\right)$ be matrix factorizations of $f$ over $R$ and $\mathcal{F}=\Phi_{\mathrm{Hmf}}(\mathcal{E})$ and $\mathcal{F}^{\prime}=\Phi_{\mathrm{Hmf}}\left(\mathcal{E}^{\prime}\right)$ be their push-forwards. Then one has

$$
\operatorname{Hom}\left(\mathcal{F}, \mathcal{F}^{\prime}\right) \cong \operatorname{Hom}\left(\mathcal{E}, \mathcal{E}^{\prime}\right) \oplus \operatorname{Hom}\left(\mathcal{E}^{\prime}, \mathcal{E}(r+h-a)\right)^{\vee}[-d] .
$$

Proof. An element of $\operatorname{Hom}\left(\mathcal{F}, \mathcal{F}^{\prime}\right)$ is represented by a pair $(\alpha, \beta)$ of morphisms

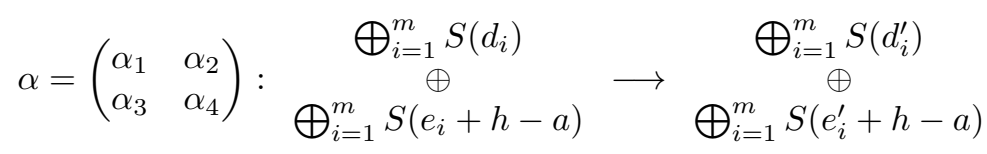

and

$$
\beta=\left(\begin{array}{ll}
\beta_{1} & \beta_{2} \\
\beta_{3} & \beta_{4}
\end{array}\right): \begin{gathered}
\bigoplus_{i=1}^{m} S\left(e_{i}\right) \\
\bigoplus_{i=1}^{m} S\left(d_{i}-a\right)
\end{gathered} \longrightarrow \begin{gathered}
\bigoplus_{i=1}^{m} S\left(e_{i}^{\prime}\right) \\
\bigoplus_{i=1}^{m} S\left(d_{i}^{\prime}-a\right)
\end{gathered}
$$


making the diagram

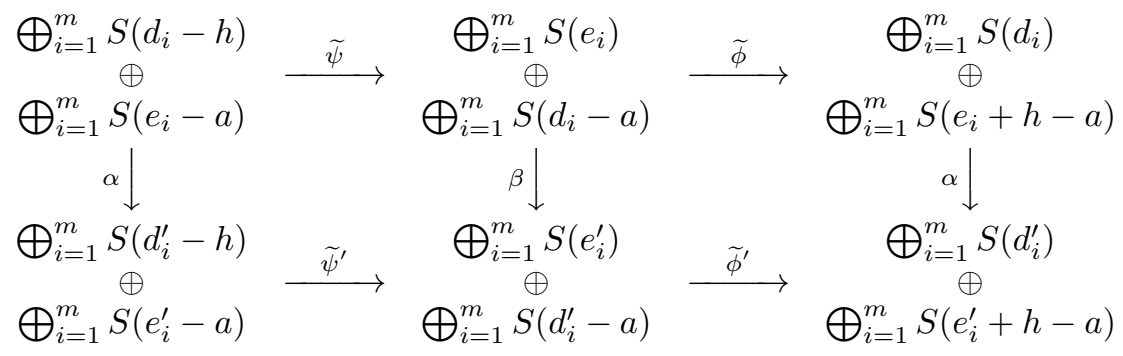

commute: $\alpha \circ \widetilde{\phi}=\widetilde{\phi}^{\prime} \circ \beta$ and $\beta \circ \widetilde{\psi}=\widetilde{\psi}^{\prime} \circ \alpha$. The former condition can be written explicitly as the equality

$$
\left(\begin{array}{cc}
\alpha_{1} \phi-\alpha_{2} g & \alpha_{1} w+\alpha_{2} \psi \\
\alpha_{3} \phi-\alpha_{4} g & \alpha_{3} w+\alpha_{4} \psi
\end{array}\right)=\left(\begin{array}{cc}
\phi^{\prime} \beta_{1}+w \beta_{3} & \phi^{\prime} \beta_{2}+w \beta_{4} \\
-g \beta_{1}+\psi^{\prime} \beta_{3} & -g \beta_{2}+\psi^{\prime} \beta_{4}
\end{array}\right)
$$

where

$$
\alpha \circ \widetilde{\phi}=\left(\begin{array}{cc}
\alpha_{1} & \alpha_{2} \\
\alpha_{3} & \alpha_{4}
\end{array}\right)\left(\begin{array}{cc}
\phi & w \\
-g & \psi
\end{array}\right)=\left(\begin{array}{cc}
\alpha_{1} \phi-\alpha_{2} g & \alpha_{1} w+\alpha_{2} \psi \\
\alpha_{3} \phi-\alpha_{4} g & \alpha_{3} w+\alpha_{4} \psi
\end{array}\right)
$$

and

$$
\widetilde{\phi}^{\prime} \circ \beta=\left(\begin{array}{cc}
\phi^{\prime} & w \\
-g & \psi^{\prime}
\end{array}\right)\left(\begin{array}{cc}
\beta_{1} & \beta_{2} \\
\beta_{3} & \beta_{4}
\end{array}\right)=\left(\begin{array}{cc}
\phi^{\prime} \beta_{1}+w \beta_{3} & \phi^{\prime} \beta_{2}+w \beta_{4} \\
-g \beta_{1}+\psi^{\prime} \beta_{3} & -g \beta_{2}+\psi^{\prime} \beta_{4}
\end{array}\right),
$$

and similarly as the equality

$$
\left(\begin{array}{cc}
\beta_{1} \psi+\beta_{2} g & -\beta_{1} w+\beta_{2} \phi \\
\beta_{3} \psi+\beta_{4} g & -\beta_{3} w+\beta_{4} \phi
\end{array}\right)=\left(\begin{array}{cc}
\psi^{\prime} \alpha_{1}-w \alpha_{3} & \psi^{\prime} \alpha_{2}-w \alpha_{4} \\
g \alpha_{1}+\phi^{\prime} \alpha_{3} & g \alpha_{2}+\phi^{\prime} \alpha_{4}
\end{array}\right)
$$

where

$$
\beta \circ \widetilde{\psi}=\left(\begin{array}{cc}
\beta_{1} & \beta_{2} \\
\beta_{3} & \beta_{4}
\end{array}\right)\left(\begin{array}{cc}
\psi & -w \\
g & \phi
\end{array}\right)=\left(\begin{array}{cc}
\beta_{1} \psi+\beta_{2} g & -\beta_{1} w+\beta_{2} \phi \\
\beta_{3} \psi+\beta_{4} g & -\beta_{3} w+\beta_{4} \phi
\end{array}\right)
$$

and

$$
\widetilde{\psi}^{\prime} \circ \alpha=\left(\begin{array}{cc}
\psi^{\prime} & -w \\
g & \phi^{\prime}
\end{array}\right)\left(\begin{array}{cc}
\alpha_{1} & \alpha_{2} \\
\alpha_{3} & \alpha_{4}
\end{array}\right)=\left(\begin{array}{cc}
\psi^{\prime} \alpha_{1}-w \alpha_{3} & \psi^{\prime} \alpha_{2}-w \alpha_{4} \\
g \alpha_{1}+\phi^{\prime} \alpha_{3} & g \alpha_{2}+\phi^{\prime} \alpha_{4}
\end{array}\right)
$$

for the latter. Two morphisms $(\alpha, \beta)$ and $\left(\alpha^{\prime}, \beta^{\prime}\right)$ are homotopic if there exist morphisms

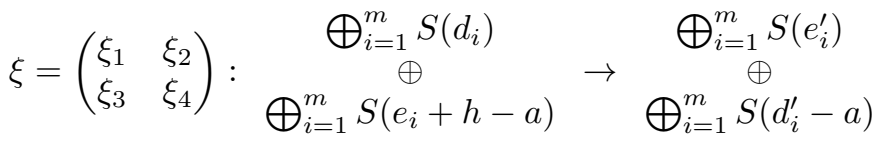

and

$$
\eta=\left(\begin{array}{ll}
\eta_{1} & \eta_{2} \\
\eta_{3} & \eta_{4}
\end{array}\right): \begin{gathered}
\bigoplus_{i=1}^{m} S\left(e_{i}\right) \\
\bigoplus_{i=1}^{m} S\left(d_{i}-a\right)
\end{gathered} \rightarrow \begin{aligned}
& \bigoplus_{i=1}^{m} S\left(d_{i}^{\prime}-h\right) \\
& \bigoplus_{i=1}^{m} S\left(e_{i}^{\prime}-a\right)
\end{aligned}
$$

satisfying

$$
\alpha-\alpha^{\prime}=\widetilde{\phi}^{\prime} \circ \xi+\eta \circ \widetilde{\psi}
$$

and

$$
\beta-\beta^{\prime}=\widetilde{\psi}^{\prime} \circ \eta+\xi \circ \widetilde{\phi}
$$


The terms on the right hand sides can be explicitly written as

$$
\begin{gathered}
\widetilde{\phi}^{\prime} \circ \xi=\left(\begin{array}{cc}
\phi^{\prime} & w \\
-g & \psi^{\prime}
\end{array}\right)\left(\begin{array}{cc}
\xi_{1} & \xi_{2} \\
\xi_{3} & \xi_{4}
\end{array}\right)=\left(\begin{array}{cc}
\phi^{\prime} \xi_{1}+w \xi_{3} & \phi^{\prime} \xi_{2}+w \xi_{4} \\
-g \xi_{3}+\psi^{\prime} \xi_{3} & -g \xi_{2}+\psi^{\prime} \xi_{4}
\end{array}\right), \\
\eta \circ \widetilde{\psi}=\left(\begin{array}{cc}
\eta_{1} & \eta_{2} \\
\eta_{3} & \eta_{4}
\end{array}\right)\left(\begin{array}{cc}
\psi & -w \\
g & \phi
\end{array}\right)=\left(\begin{array}{cc}
\eta_{1} \psi+\eta_{2} g & -\eta_{1} w+\eta_{2} \phi \\
\eta_{3} \psi+\eta_{4} g & -\eta_{3} w+\eta_{4} \phi
\end{array}\right), \\
\widetilde{\psi}^{\prime} \circ \eta=\left(\begin{array}{cc}
\psi^{\prime} & -w \\
g & \phi^{\prime}
\end{array}\right) \circ\left(\begin{array}{cc}
\eta_{1} & \eta_{2} \\
\eta_{3} & \eta_{4}
\end{array}\right)=\left(\begin{array}{cc}
\psi^{\prime} \eta_{1}-w \eta_{3} & \psi^{\prime} \eta_{2}-w \eta_{4} \\
g \eta_{1}+\phi^{\prime} \eta_{3} & g \eta_{2}+\phi^{\prime} \eta_{4}
\end{array}\right), \\
\xi \circ \widetilde{\phi}=\left(\begin{array}{cc}
\xi_{1} & \xi_{2} \\
\xi_{3} & \xi_{4}
\end{array}\right)\left(\begin{array}{cc}
\phi & w \\
-g & \psi
\end{array}\right)=\left(\begin{array}{ll}
\xi_{1} \phi-\xi_{2} g & \xi_{1} w+\xi_{2} \psi \\
\xi_{3} \phi-\xi_{4} g & \xi_{3} w+\xi_{4} \psi
\end{array}\right) .
\end{gathered}
$$

Note that one can remove $w$-dependence of $\alpha_{1}$ by choosing $\xi_{3}$ in a suitable way and achieve $\alpha_{1} \in \operatorname{Mat}_{m}(R)$. Similarly, $w$-dependence of $\alpha_{2}, \beta_{1}$ and $\beta_{2}$ can be removed by choosing $\xi_{4}, \eta_{3}$ and $\eta_{4}$ respectively in a suitable way. Moreover, these operations of removing $w$-dependence can be performed independently so that one can achieve $\alpha_{1}, \alpha_{2}, \beta_{1}, \beta_{2} \in \operatorname{Mat}_{m}(R)$ simultaneously.

Now the $(1,1)$-component of (4.1) gives the equation

$$
\alpha_{1} \phi-\alpha_{2} g=\phi^{\prime} \beta_{1}+w \beta_{3} .
$$

Since $\alpha_{1}, \beta_{1}, \phi, \phi^{\prime} \in \operatorname{Mat}_{m}(R)$ and $\alpha_{2} g, w \beta_{3} \in w \operatorname{Mat}_{m}(S)$ in the direct sum decomposition $\operatorname{Mat}_{m}(S)=\operatorname{Mat}_{m}(R) \oplus w \operatorname{Mat}_{m}(S)$, one obtains

$$
\alpha_{1} \phi=\phi^{\prime} \beta_{1} \quad \text { and } \quad \beta_{3}=-\frac{g}{w} \alpha_{2} .
$$

Similarly, from the (1,2)-component of (4.1), one obtains

$$
\alpha_{1} w+\alpha_{2} \psi=\phi^{\prime} \beta_{2}+w \beta_{4},
$$

which, together with $\alpha_{2}, \beta_{2}, \psi, \phi^{\prime} \in \operatorname{Mat}_{m}(R)$, implies

$$
\alpha_{2} \psi=\phi^{\prime} \beta_{2} \quad \text { and } \quad \beta_{4}=\alpha_{1} .
$$

The same argument for $(1,1)$ - and $(1,2)$-components of (4.2) gives

$$
\beta_{1} \psi=\psi^{\prime} \alpha_{1} \quad \text { and } \quad \alpha_{3}=-\frac{g}{w} \beta_{2}
$$

and

$$
\beta_{2} \phi=\psi^{\prime} \alpha_{2} \quad \text { and } \quad \alpha_{4}=\beta_{1}
$$

respectively. This determines $\alpha$ and $\beta$ completely from $\left(\alpha_{1}, \alpha_{2}, \beta_{1}, \beta_{2}\right)$ satisfying

$$
\alpha_{1} \phi=\phi^{\prime} \beta_{1}, \quad \alpha_{2} \psi=\phi^{\prime} \beta_{2}, \quad \beta_{1} \psi=\psi^{\prime} \alpha_{1}, \quad \beta_{2} \phi=\psi^{\prime} \alpha_{2} .
$$

Other components of (4.1) and (4.2) are automatically satisfied for $\alpha$ and $\beta$ satisfying (4.7), (4.8), (4.9), and (4.10). This shows that any element of $\operatorname{Hom}\left(\mathcal{F}, \mathcal{F}^{\prime}\right)$ can be represented by a pair $(\alpha, \beta)$ of morphisms coming from a representative $\left(\alpha_{1}, \beta_{1}\right)$ of $\operatorname{Hom}\left(\mathcal{E}, \mathcal{E}^{\prime}\right)$ and a representative $\left(\alpha_{2}, \beta_{2}\right)$ of $\operatorname{Hom}\left(\mathcal{E}[1](-a), \mathcal{E}^{\prime}\right)$.

If two morphisms from $\mathcal{F}$ to $\mathcal{F}^{\prime}$ represented by pairs $(\alpha, \beta)$ and $\left(\alpha^{\prime}, \beta^{\prime}\right)$ coming from representatives $\left(\alpha_{1}, \beta_{1}\right)$ and $\left(\alpha_{1}^{\prime}, \beta_{1}^{\prime}\right)$ of $\operatorname{Hom}\left(\mathcal{E}, \mathcal{E}^{\prime}\right)$ and representatives $\left(\alpha_{2}, \beta_{2}\right)$ and $\left(\alpha_{2}^{\prime}, \beta_{2}^{\prime}\right)$ of $\operatorname{Hom}\left(\mathcal{E}[1](-a), \mathcal{E}^{\prime}\right)$ are homotopic, then one has morphisms $\xi$ and $\eta$ 
as in (4.3) and (4.4) satisfying (4.5) and (4.6). Let $\bar{\xi}_{1}$ and $\bar{\eta}_{1}$ be the $\operatorname{Mat}_{m}(R)$ components of $\xi_{1}$ and $\eta_{1}$ in the direct sum decomposition $\operatorname{Mat}_{m}(S)=\operatorname{Mat}_{m}(R) \oplus$ $w \operatorname{Mat}_{m}(S)$. Then the $\operatorname{Mat}_{m}(R)$ component of the upper-left $m \times m$-block of (4.5) gives

$$
\alpha_{1}-\alpha_{1}^{\prime}=\phi^{\prime} \bar{\xi}_{1}+\bar{\eta}_{1} \psi
$$

and that of (4.6) gives

$$
\beta_{1}-\beta_{1}^{\prime}=\psi^{\prime} \bar{\eta}_{1}+\bar{\xi}_{1} \phi
$$

(4.12) and (4.13) show that $\left(\bar{\xi}_{1}, \bar{\eta}_{1}\right)$ gives a homotopy between $\left(\alpha_{1}, \beta_{1}\right)$ and $\left(\alpha_{1}^{\prime}, \beta_{1}^{\prime}\right)$ as matrix factorizations of $f$ over $R$. Similarly, the $\operatorname{Mat}_{m}(R)$ components of the upper-right $m \times m$-blocks of (4.5) and (4.6) give

$$
\alpha_{2}-\alpha_{2}^{\prime}=\phi^{\prime} \bar{\xi}_{2}+\bar{\eta}_{2} \phi
$$

and

$$
\beta_{2}-\beta_{2}^{\prime}=\psi^{\prime} \bar{\eta}_{2}+\bar{\xi}_{2} \psi
$$

which show that $\left(\alpha_{2}, \beta_{2}\right)$ and $\left(\alpha_{2}^{\prime}, \beta_{2}^{\prime}\right)$ are homotopic as matrix factorizations of $f$ over $R$. It follows that one has an isomorphism

$$
\operatorname{Hom}\left(\mathcal{F}, \mathcal{F}^{\prime}\right) \cong \operatorname{Hom}\left(\mathcal{E}, \mathcal{E}^{\prime}\right) \oplus \operatorname{Hom}\left(\mathcal{E}[1](-a), \mathcal{E}^{\prime}\right)
$$

of spaces of morphisms. Graded Auslander-Reiten duality AR87 implies the Serre duality

$$
\operatorname{Hom}(\mathcal{M}, \mathcal{N}) \cong \operatorname{Hom}(\mathcal{N}, \mathcal{M}(r+h)[d-1])^{\vee}
$$

in the graded stable derived category of $\bar{R}$ [T , Corollary 2.5], so that

$$
\operatorname{Hom}\left(\mathcal{E}[1](-a), \mathcal{E}^{\prime}\right) \cong \operatorname{Hom}\left(\mathcal{E}^{\prime}, \mathcal{E}(r+h-a)\right)^{\vee}[-d],
$$

and Proposition 4.1 is proved.

\section{ACKNOWLEDGMENTS}

The author thanks Yukinobu Toda for pointing out an error in (1.1) in the earlier version. This research was supported by Grant-in-Aid for Young Scientists No. 24740043.

\section{REFERENCES}

[AR87] Maurice Auslander and Idun Reiten, Almost split sequences for Z-graded rings, (Lambrecht, 1985), Lecture Notes in Math., vol. 1273, Springer, Berlin, 1987, pp. 232243, DOI 10.1007/BFb0078847. MR915178 (89b:13031)

[BH93] Per Berglund and Tristan Hübsch, A generalized construction of mirror manifolds, Nuclear Phys. B 393 (1993), no. 1-2, 377-391, DOI 10.1016/0550-3213(93)90250-S. MR 1214325 (94k:14031)

[Bor] Lev A. Borisov, Berglund-Hübsch mirror symmetry via vertex algebras, Comm. Math. Phys. 320 (2013), no. 1, 73-99. MR3046990

[Buc87] Ragnar-Olaf Buchweitz, Maximal Cohen-Macaulay modules and Tate-cohomology over Gorenstein rings, 1987. Available at https://tspace.library.utoronto.ca/handle/ $1807 / 16682$.

[CR11] Alessandro Chiodo and Yongbin Ruan, LG/CY correspondence: the state space isomorphism, Adv. Math. 227 (2011), no. 6, 2157-2188, DOI 10.1016/j.aim.2011.04.011. MR2807086 (2012g:14069) 
[Eis80] David Eisenbud, Homological algebra on a complete intersection, with an application to group representations, Trans. Amer. Math. Soc. 260 (1980), no. 1, 35-64, DOI 10.2307/1999875. MR570778(82d:13013)

[FU] Masahiro Futaki and Kazushi Ueda, Homological mirror symmetry for singularities of type D, Math. Z. 273 (2013), no. 3-4, 633-652, DOI 10.1007/s00209-012-1024-x. MR3030671

[FU11] Masahiro Futaki and Kazushi Ueda, Homological mirror symmetry for Brieskorn-Pham singularities, Selecta Math. (N.S.) 17 (2011), no. 2, 435-452, DOI 10.1007/s00029-0100055-6. MR2803848(2012e:14083)

[Hap91] Dieter Happel, On Gorenstein algebras (Bielefeld, 1991), Progr. Math., vol. 95, Birkhäuser, Basel, 1991, pp. 389-404. MR1112170 (92k:16022)

[IT] Osamu Iyama and Ryo Takahashi, Tilting and cluster tilting for quotient singularities, Math. Ann. 356 (2013), no. 3, 1065-1105. MR3063907

[KMU12] Masanori Kobayashi, Makiko Mase, and Kazushi Ueda, A note on exceptional unimodal singularities and K3 surfaces, Internat. Math. Res. Notices 2013, no. 7. MR3044454

[Kon95] Maxim Kontsevich, Homological algebra of mirror symmetry, Proc. ICM, Vol. 2 (Zürich, 1994), Birkhäuser, Basel, 1995, pp. 120-139. MR1403918 (97f:32040)

[Kra] Marc Krawitz, FJRW rings and Landau-Ginzburg mirror symmetry, University of Michigan, 2010, 67 pp., ProQuest LLC. MR2801653

[Kra05] Henning Krause, The stable derived category of a Noetherian scheme, Compos. Math. 141 (2005), no. 5, 1128-1162, DOI 10.1112/S0010437X05001375. MR.2157133 (2006e:18019)

[NU12] Yuichi Nohara and Kazushi Ueda, Homological mirror symmetry for the quintic 3-fold, Geom. Topol. 16 (2012), no. 4, 1967-2001. MR2975297

[Or104] D. O. Orlov, Triangulated categories of singularities and D-branes in Landau-Ginzburg models (Russian, with Russian summary), Tr. Mat. Inst. Steklova 246 (2004, Algebr. Geom. Metody, Svyazi i Prilozh.), 240-262; English transl., Proc. Steklov Inst. Math. 3 (246) (2004), 227-248. MR2101296 (2006i:81173)

[Orl09] Dmitri Orlov, Derived categories of coherent sheaves and triangulated categories of singularities, Algebra, arithmetic, and geometry: In honor of Yu. I. Manin. Vol. II, Progr. Math., vol. 270, Birkhäuser Boston Inc., Boston, MA, 2009, pp. 503-531, DOI 10.1007/978-0-8176-4747-6_16. MR2641200 (2011c:14050)

[Sei02] Paul Seidel, Fukaya categories and deformations (Beijing, 2002), Higher Ed. Press, Beijing, 2002, pp. 351-360. MR.1957046 (2004a:53110)

[Sei08] Paul Seidel, Fukaya categories and Picard-Lefschetz theory, Zurich Lectures in Advanced Mathematics, European Mathematical Society (EMS), Zürich, 2008. MR2441780

[Sei11] Paul Seidel, Homological mirror symmetry for the quartic surface, math.AG/0310414, 2011.

[She11] Nick Sheridan, On the homological mirror symmetry conjecture for pairs of pants, J. Differential Geom. 89 (2011), no. 2, 271-367. MR2863919 (2012m:53196)

[Tak10] Atsushi Takahashi, Weighted projective lines associated to regular systems of weights of dual type, New developments in algebraic geometry, integrable systems and mirror symmetry (RIMS, Kyoto, 2008), Adv. Stud. Pure Math., vol. 59, Math. Soc. Japan, Tokyo, 2010, pp. 371-388. MR.2683215(2012b:14080)

Department of Mathematics, Graduate School of Science, Osaka University, Machikaneyama 1-1, TOYONAKA, Osaka, 560-0043, JAPAN

E-mail address: kazushi@math.sci.osaka-u.ac.jp 